Article

\title{
The Lethal(2)-Essential-for-Life [L(2)EFL] Gene Family Modulates Dengue Virus Infection in Aedes aegypti
}

\author{
Lucky R. Runtuwene ${ }^{1,2,3, *,+}$, Shuichi Kawashima ${ }^{4,+}$, Victor D. Pijoh ${ }^{5}$, Josef S. B. Tuda ${ }^{5}$, \\ Kyoko Hayashida ${ }^{6}$, Junya Yamagishi ${ }^{6,7}$, Chihiro Sugimoto ${ }^{6}$, Shoko Nishiyama ${ }^{8}$, \\ Michihito Sasaki ${ }^{9}\left(\mathbb{D}\right.$, Yasuko Orba ${ }^{9}\left(\mathbb{D}\right.$, Hirofumi Sawa $9{ }^{(D)}$, Tomohiko Takasaki ${ }^{10,11}(\mathbb{D}$, \\ Anthony A. James ${ }^{12}$, Takashi Kobayashi ${ }^{1}$ and Yuki Eshita $1,13,14,15, *$ (D) \\ 1 Department of Infectious Disease Control, Faculty of Medicine, Oita University, Oita 879-5593, Japan; \\ takashik@oita-u.ac.jp \\ 2 Department of Computational Biology, Graduate School of Frontier Sciences, University of Tokyo, \\ 5-1-5 Kashiwanoha, Kashiwa, Chiba 277-8562, Japan \\ 3 AIDS Research Centre, National Institute of Infectious Diseases, Tokyo 162-8640, Japan \\ 4 Database Center for Life Science, Joint Support-Center for Data Science Research, Research Organization of \\ Information and Systems, 178-4-4 Wakashiba, Kashiwa, Chiba 277-0871, Japan; kwsm@dbcls.roia.ac.jp \\ 5 Faculty of Medicine, Sam Ratulangi University, Kampus Unsrat, Bahu Manado 95-115, Indonesia; \\ victorpijoh@gmail.com (V.D.P.); jsbtuda@yahoo.com (J.S.B.T.) \\ 6 Division of Collaboration and Education, Research Center for Zoonosis Control, Hokkaido University, \\ North 20, West 10 Kita-ku, Sapporo, Hokkaido 001-0020, Japan; kyouko-h@czc.hokudai.ac.jp (K.H.); \\ junya@czc.hokudai.ac.jp (J.Y.); sugimoto@czc.hokudai.ac.jp (C.S.) \\ 7 Global Station for Zoonosis Control, GI-CoRE, Hokkaido University, North 20, West 10 Kita-ku, Sapporo, \\ Hokkaido 001-0020, Japan \\ 8 Laboratory of Zoonotic Diseases, Faculty of Applied Biological Sciences, Gifu University, 1-1 Yanagido, \\ Gifu-shi, Gifu 501-1193, Japan; shnishiy@gifu-u.ac.jp \\ 9 Division of Molecular Pathobiology, Research Center for Zoonosis Control, Hokkaido University, \\ North 20, West 10 Kita-ku, Sapporo, Hokkaido 001-0020, Japan; m-sasaki@czc.hokudai.ac.jp (M.S.); \\ orbay@czc.hokudai.ac.jp (Y.O.); h-sawa@czc.hokudai.ac.jp (H.S.) \\ 10 National Institute of Infectious Diseases, Tokyo 162-8640, Japan; dengue3124@yahoo.co.jp \\ 11 Kanagawa Prefectural Institute of Public Health, Kanagawa 253-0087, Japan \\ 12 Departments of Microbiology \& Molecular Genetics and Molecular Biology \& Biochemistry, \\ University of California, Irvine, CA 92697, USA; aajames@uci.edu \\ 13 Departments of Medical Entomology, Faculty of Tropical Medicine, Mahidol University, \\ 420/6 Ratchawithi Road, Rajathewi, Bangkok 10400, Thailand \\ 14 Research Institute for Microbial Diseases, Osaka University, 3-1 Yamadaoka, Suita, Osaka 565-0871, Japan \\ 15 Hokudai Center for Zoonosis Control in Zambia, Research Center for Zoonosis Control, \\ Hokkaido University, North 20, West 10 Kita-ku, Sapporo, Hokkaido 001-0020, Japan \\ * Correspondence: luckyron@niid.go.jp (L.R.R.); yeshita@czc.hokudai.ac.jp (Y.E.) \\ $\dagger$ These authors contributed equally to this work.
}

Received: 2 September 2020; Accepted: 8 October 2020; Published: 12 October 2020

check for updates

\begin{abstract}
Efforts to determine the mosquito genes that affect dengue virus replication have identified a number of candidates that positively or negatively modify amplification in the invertebrate host. We used deep sequencing to compare the differential transcript abundances in Aedes aegypti 14 days post dengue infection to those of uninfected A. aegypti. The gene lethal(2)-essential-for-life [l(2)efl], which encodes a member of the heat shock 20 protein (HSP20) family, was upregulated following dengue virus type 2 (DENV-2) infection in vivo. The transcripts of this gene did not exhibit differential accumulation in mosquitoes exposed to insecticides or pollutants. The induction and overexpression of l(2)efl gene products using poly(I:C) resulted in decreased DENV-2 replication in the cell line. In contrast, the RNAi-mediated suppression of $l(2)$ efl gene products resulted in enhanced
\end{abstract}


DENV-2 replication, but this enhancement occurred only if multiple $l(2) e f l$ genes were suppressed. l(2)efl homologs induce the phosphorylation of eukaryotic initiation factor $2 \alpha$ (eIF2 $\alpha$ ) in the fruit fly Drosophila melanogaster, and we confirmed this finding in the cell line. However, the mechanism by which $l(2) e f l$ phosphorylates eIF $2 \alpha$ remains unclear. We conclude that $l(2) e f l$ encodes a potential anti-dengue protein in the vector mosquito.

Keywords: lethal(2)-essential-for-life; dengue; Aedes aegypti

\section{Introduction}

Aedes aegypti is an important vector of human disease pathogens, and it transmits dengue viruses that cause a spectrum of diseases that are of increasing concern as emerging infections [1,2]. The vector mosquito is hematophagous and usually becomes infected by dengue virus while feeding on the blood of infected individuals. After proliferation in the midgut and dispersal through the mosquito body to the salivary glands, the virus is transmitted to uninfected persons during subsequent blood feedings [3]. Breaking the transmission cycle is important, as effective vaccines and specific preventative and therapeutic drugs are not yet available [4-6]. The only approved dengue vaccine has been reviewed as unsafe for seronegative individuals at the time of vaccination [7], which is a drawback in dengue prevention. Traditional methods to control transmission include the avoidance of mosquito bites using repellents and suppressing mosquito populations with insecticides and breeding site removal [2]. However, insecticide resistance and the ineffectiveness of community-based source reduction make it impossible to sustain breaks in transmission [8]. The development of transgenic mosquitoes for either population suppression (genetic analog to an insecticide) or population modification (rendering the mosquito genetically incapable of transmitting the viruses) are among the proposals for novel control methods $[9,10]$.

Scientists have tried to elaborate the complex relationships between dengue viruses and their vectors. Experiments using next-generation sequencing to perform a comprehensive analysis of gene expression following dengue infection have been carried out [11,12]. Researchers have predicted protein interactions between dengue viruses and A. aegypti using computer models [13,14]. Protein profiles of dengue-infected $A$. aegypti also have been published [15]. The mosquito transcriptome profiles in response to dengue infection have been studied using microarrays in cells [16], dissected organs [17,18], or whole-body preparations [11]. Specific genes, such as those related to immunity or innate immune pathways also have been investigated $[19,20]$.

We used deep sequencing to analyze the differential transcript abundances between dengue-infected and uninfected A. aegypti. In contrast to previous studies that used young mice or viruses cultured in cells, we used adult immunocompetent mice for the challenge assay [21]. We infected the Liverpool strain of $A$. aegypti, for which the complete genome sequence is available [22], with dengue virus type 2 (DENV-2) Southeast Asian type, an isolate that has the potential to cause severe disease [23,24]. We identified a family of genes, including lethal(2)-essential-for-life [l(2)efl], which encodes a product belonging to the heat shock protein 20 (HSP20) family and whose transcripts are more abundant in infected mosquitoes than in uninfected controls. The induction or inhibition of the gene and family members in vitro results in decreased and increased DENV-2 replication, respectively.

\section{Results and Discussion}

\subsection{Mosquito Infection with DENV-2 Using Direct Feeding}

Mosquitoes were infected by allowing them to feed to repletion on adult immunocompetent mice infected with DENV-2, as previously reported [21]. We used 5- to 9-day-old mosquitoes to increase the blood feeding rate. At this age, the mosquitoes had reached the second stage of ovarian 
development and would physiologically seek blood. We also performed the feeding in the late afternoon to coincide with the maximum circadian feeding activity. RT-PCR screening detected the DENV-2 genome in samples of mosquito legs collected at 6, 10, and $14 \mathrm{dpi}$, but not in 2 dpi mosquitoes (Figure 1A). These results are consistent with previous reports that demonstrated that the DENV-2 antigen is detected in the A. aegypti Chetumal strain in the trachea and salivary glands at 2 and 4 dpi, respectively [25]. However, the dissemination timing varies among mosquito strains, as salivary gland infection of Rex-D mosquitoes was not observed until $10 \mathrm{dpi}$. One of five mosquitoes at $10 \mathrm{dpi}$ in the present study did not show any viral genome in its legs, although the RT-PCR of its carcass showed the presence of the DENV-2 genome (Figure 1B), thereby presenting evidence of a midgut barrier in this specific mosquito strain. Furthermore, one mosquito was not infected with the dengue virus, as shown by the absence of the DENV-2 genome in its carcass. As many as $99.6-100 \%$ of the female mosquitoes in each cage clearly fed on infected mice. Therefore, we did not remove the nonfeeding mosquitoes due to safety reasons. Moreover, we found that the DENV-2 kinetics in A. aegypti were stable for 2 to 14 days (Figure 1C).
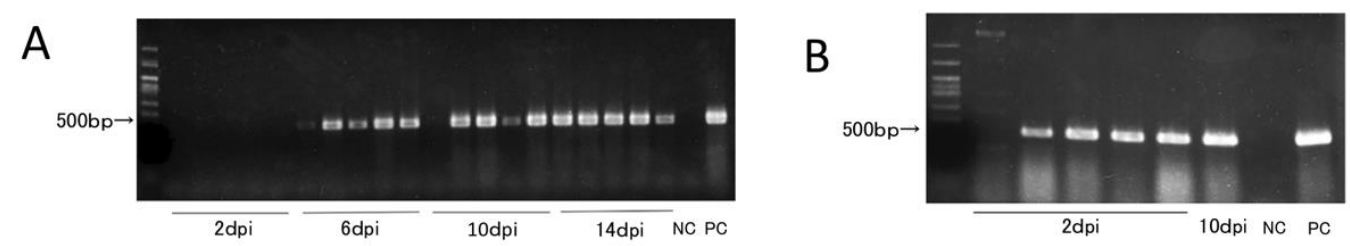

$$
\text { C }
$$

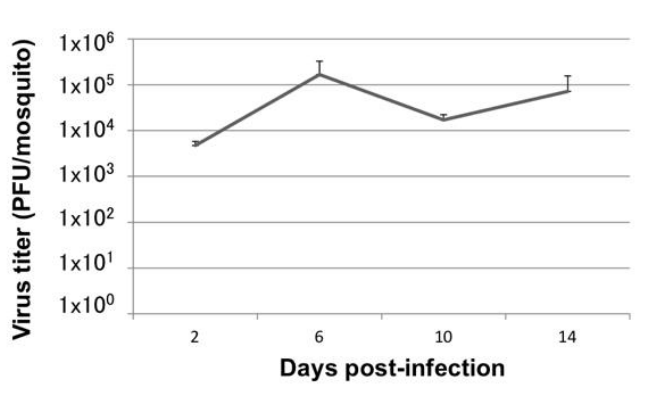

D
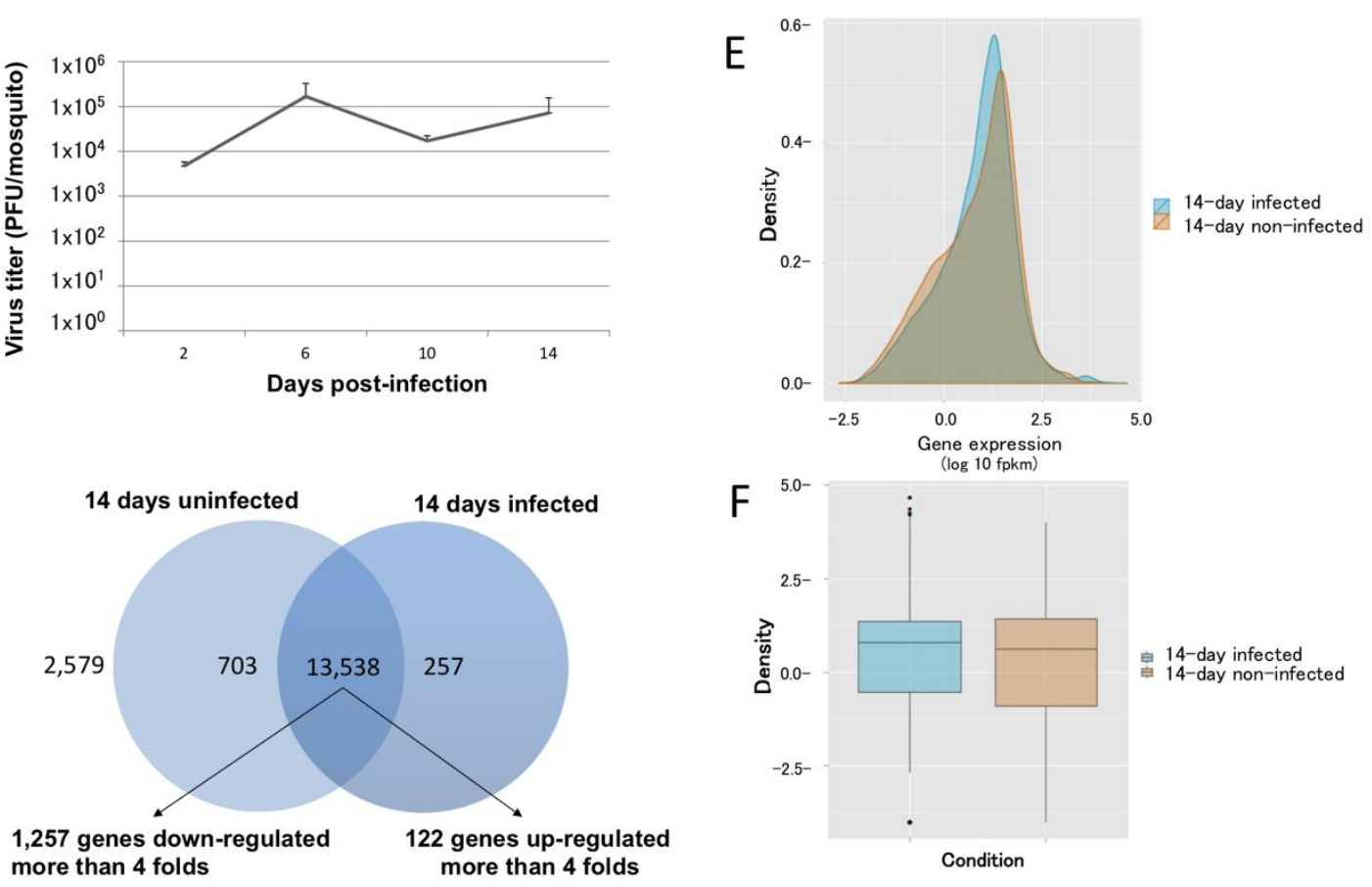

Figure 1. Temporal, spatial, and quantitative dynamics of dengue virus dissemination in Aedes aegypti. (A) Dengue virus (DENV) dissemination at 2, 6, 8, and 10 days post-infection (dpi) ( $\mathrm{N}=5$ for each time point). (B) At 2 dpi, infected mosquitoes had DENV in their carcasses. One mosquito was not infected, and one $10 \mathrm{dpi}$ mosquito had an apparent midgut escape barrier that prevented DENV dissemination. (C) The DENV titer in infected mosquitoes was stable for up to $14 \mathrm{dpi}(\mathrm{N}=3)$. (D) As many as 13,538 genes were affected by DENV infection at 14 dpi, with 1257 and 122 genes showing decreased and increased transcript abundance, respectively, by more than 4 -fold. As many as 2579 genes had products that showed no differential abundance. (E,F) Density and box plots of the overlapped transcript accumulation between 14-day-infected and uninfected mosquitoes. NC: negative control; PC: positive control. 


\subsection{Sequencing Data}

A total of 131,774,320 reads were mapped to the reference genome using Bowtie v2.2 and TopHat v2.1, with an efficiency of $90.5 \%$ and $95.1 \%$ for the infected and uninfected groups, respectively (Table 1). It is possible that a few uninfected mosquitoes were included in the infected population, but the fraction $(\leq 1 / 20$ as determined above) was anticipated to be too small to affect the results. The differential transcript abundance of 10 selected loci (histone H4 [AAEL003689], lethal(2)-essential-for-life [AAEL013338], 4-nitrophenyl phosphatase [AAEL007097], cdc6 [AAEL010855], cactus [AAEL000709], antibacterial peptide [AAEL004233], hypothetical protein [AAEL004851], vago [AAEL000200], zinc carboxypeptidase [AAEL001863], carboxypeptidase [AAEL010766]) identified by RNA-seq were confirmed by qRT-PCR. These 10 loci exhibited a Spearman Rho's R of $0.79394(p<0.01)$ between the two assays (Table 2).

Table 1. RNA-seq generated more than 60 million reads in both groups. Among these reads, Bowtie v2.2 and TopHat v2.1 mapped at least $87.7 \%$ of the reads to the reference genome. At least one successfully aligned read was required for inclusion.

\begin{tabular}{ccccc}
\hline Sample Source & Replicate & $\begin{array}{c}\text { Number of } \\
\text { Reads Processed }\end{array}$ & $\begin{array}{c}\text { Number of Reads with } \geq \mathbf{1} \text { Reported } \\
\text { Alignment (Percent of Total Reads) }\end{array}$ & $\begin{array}{c}\text { Number of Reads that Failed to } \\
\text { Align (Percent of Total Reads) }\end{array}$ \\
\hline \multirow{2}{*}{ 14 days noninfected } & 1 & $39,174,903$ & $37,360,756(95.4 \%)$ & $1,814,147(4.6 \%)$ \\
\cline { 2 - 5 } & 2 & $30,866,781$ & $29,259,296(94.8)$ & $1,607,485(5.2 \%)$ \\
\hline \multirow{2}{*}{ 14 days infected } & 1 & $27,119,695$ & $23,790,556(87.7 \%)$ & $3,849,139(12.3 \%)$ \\
\hline
\end{tabular}

Table 2. Real-time PCR was used to validate the RNA-seq data. We randomly selected 10 genes and confirmed their expression in a pool of 10 mosquitoes. The RNA-seq and qRT-PCR data were obtained from two different mosquito populations. Compared to the RNA-seq data, the qRT-PCR data does not show large discrepancies in the abundances. The RNA-seq and qRT-PCR data are correlated statistically with a Spearman Rho's R of $0.79394(p<0.01)$ between the two assays. qRT-PCR values are the average of two biological replicates.

\begin{tabular}{|c|c|c|c|c|c|c|}
\hline \multirow{3}{*}{ Gene ID } & \multirow{3}{*}{ Gene Annotation } & \multicolumn{3}{|c|}{ RNA-seq } & \multirow{2}{*}{\multicolumn{2}{|c|}{$\frac{\text { qRT-PCR }}{\text { Normalized Abundance Values }}$}} \\
\hline & & \multicolumn{2}{|c|}{ RPKM } & \multirow{2}{*}{$\begin{array}{c}\text { Fold } \\
\text { Changes }\end{array}$} & & \\
\hline & & Uninfected & Infected & & Uninfected & Infected \\
\hline AAEL013338 & Lethal(2)-essential-for-life & 743.143 & 7346.79 & 9.9 & 1.00 & 3.84 \\
\hline AAEL007097 & 4-nitrophenyl phosphatase & 918.192 & 6941.7 & 7.56 & 1.00 & 2.55 \\
\hline AAEL000709 & Cactus & 113.145 & 219.984 & 1.944 & 1.00 & 1.76 \\
\hline AAEL004223 & Antibacterial peptide & 17608.2 & 4622.73 & -3.8 & 1.00 & 2.20 \\
\hline AAEL004851 & Hypothetical protein & 46960.7 & 9712.4 & -4.835 & 1.00 & 1.77 \\
\hline AAEL000200 & Hypothetical protein & 464.005 & 75.3489 & -6.158 & 1.00 & 0.62 \\
\hline
\end{tabular}

\subsection{Identification of $L(2)$ efl}

Cufflinks processing showed that the transcript abundance of 13,538 genes $(\sim 79.3 \%$ of annotated genes) varied between the samples (Figure 1D, Supplementary Tables S4 and S5) with 6704 and 6834 genes with transcripts that increased or decreased, respectively. A total of 122/6704 and 1257/6834 of the genes had $>4$-fold increases or decreases, respectively. Moreover, 703 and 257 transcripts were found exclusively in uninfected or infected mosquitoes, respectively. As many as 2579 genes did not show any differential transcript accumulation. Most of the genes had overlapping expression patterns, with a trend towards upregulation between the two groups (Figure 1E,F). The majority of genes whose transcripts increased in abundance in infected mosquitos were annotated by Gene 
Ontology [26] as binding and catalytic activity proteins. Genes exhibiting decreases were annotated as having catalytic activity (Supplementary Tables S4 and S5). Collectively, histone protein-encoding loci represented $29.61 \%$ of the genes, with the transcript abundance increasing $\geq 4$-fold in infected mosquitoes. This observation is consistent with the fact that the DENV capsid protein has been shown to target the four core histones (H2A, H2B, H3, and H4) in liver cells [27], which are replaced by increasing their transcription.

Genes encoding several members of the heat shock protein 20 (HSP20) family also showed modulations in transcript accumulation. HSPs are known to be induced during stress (including heat shock, pathogen infection, heavy metal ion exposure, hypoxia, and osmotic stress), and they act as chaperones to guide misfolded proteins [28]. The A. aegypti genome includes 19 genes with the HSP20 domain. We detected increased differential transcript expression in infected mosquitoes for nine of these genes, including the lethal(2)-essential-for-life [l(2)efl] locus (Table 3).

Table 3. The heat shock protein 20 (HSP20) gene has 19 homologs in the A. aegypti genome. RNA-seq data showed that the majority of the homologs were expressed differentially compared to those in uninfected mosquitoes. Nine of these homologs, which included the $l(2)$ efl gene, were upregulated.

\begin{tabular}{|c|c|c|}
\hline Gene ID & Gene Annotation & $\begin{array}{c}\text { Abundance Level Compared to } \\
\text { Uninfected Mosquitoes (2-log-fold) }\end{array}$ \\
\hline AAEL003344 & Metaxin & 0 \\
\hline AAEL010654 & Lethal(2)-essential-for-life protein, l(2)efl & -2.51386 \\
\hline AAEL010660 & Alpha-B-crystallin, putative & -2.86544 \\
\hline AAEL010664 & Actin binding protein, putative & -0.90751 \\
\hline AAEL010667 & Lethal(2)-essential-for-life protein, l(2)efl & 0 \\
\hline AAEL010670 & Lethal(2)-essential-for-life protein, $l(2) e f l$ & 2.80795 \\
\hline AAEL013338 & Lethal(2)-essential-for-life protein, l(2)efl & 3.3054 \\
\hline AAEL013339 & AlphaA-crystallin, putative & 0.816776 \\
\hline AAEL013340 & Lethal(2)-essential-for-life protein, l(2)efl & -0.247543 \\
\hline AAEL013341 & Lethal(2)-essential-for-life protein, l(2)efl & -1.30716 \\
\hline AAEL013344 & Lethal(2)-essential-for-life protein, l(2)efl & 3.03323 \\
\hline AAEL013345 & AlphaA-crystallin, putative & 0.752978 \\
\hline AAEL013346 & Lethal(2)-essential-for-life protein, l(2)efl & 1.63413 \\
\hline AAEL013347 & Lethal(2)-essential-for-life protein, l(2)efl & -0.812587 \\
\hline AAEL013348 & Lethal(2)-essential-for-life protein, l(2)efl & 0 \\
\hline AAEL013349 & Lethal(2)-essential-for-life protein, l(2)efl & 1.00427 \\
\hline AAEL013350 & Heat shock protein $26 \mathrm{kD}$, putative & 2.43882 \\
\hline AAEL013351 & Lethal(2)-essential-for-life protein, l(2)efl & 1.48215 \\
\hline AAEL013352 & Lethal(2)-essential-for-life protein, l(2)efl & -0.581082 \\
\hline
\end{tabular}

$L(2) e f l$ is expressed in DENV-2-infected or poly(I:C)-transfected CCL-125 cells and modulates.

\subsection{DENV-2 Replication}

We infected the A. aegypti CCL-125 cell line with DENV-2 at an MOI of 5.0. Consistent with the RNA-seq data, the l(2)efl (AAEL013338) expression analyzed by RT-PCR was increased by approximately 3.25-fold by DENV-2 infection at $6 \mathrm{~h}$ post infection and reached a peak of approximately 4.75 -fold at $12 \mathrm{~h}$ post infection (Figure 2A). Poly(I:C), a synthetic dsRNA, transfection into CCL-125 cells also induced the expression of $l(2)$ efl, with the transcript abundance reaching a peak ( 1.6-fold) at $3 \mathrm{~h}$ post transfection (Figure 2B). The synthetic agent did not achieve the highest level induced by DENV-2. 
A

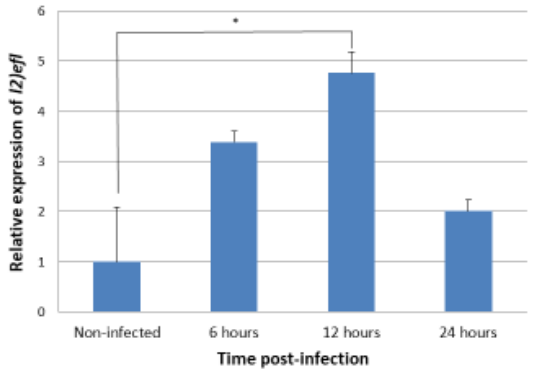

B

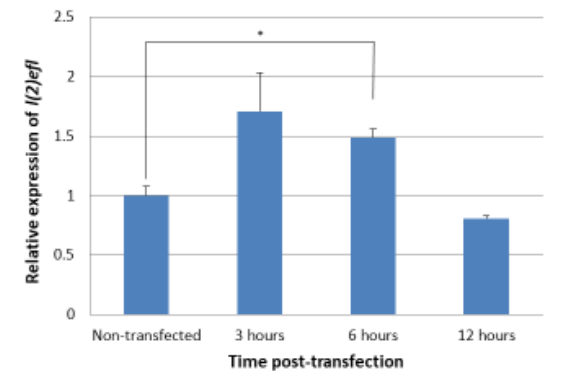

C

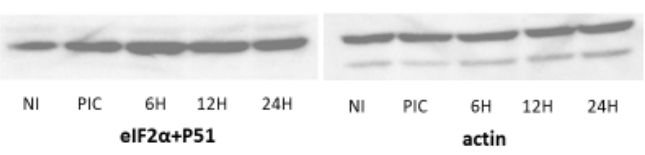

D

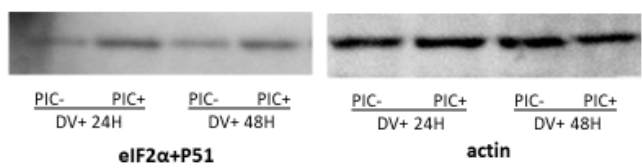

$\mathrm{E}$

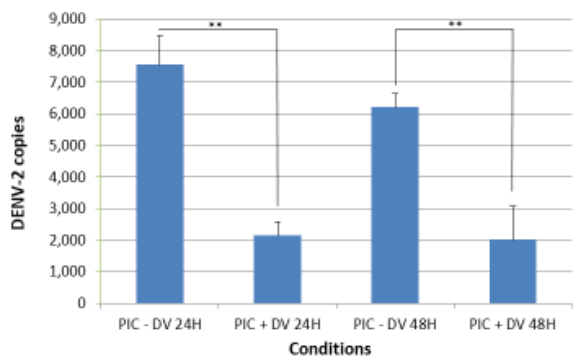

Figure 2. Dengue-virus infection increases the $l(2)$ efl expression and phosphorylates eIF2 $\alpha$ in CCL-125 cells. (A) When infected with dengue virus (DENV), $l(2)$ efl was upregulated as high as $\sim 5$-fold, with a peak at $12 \mathrm{~h}$ post infection (hpi). (B) The poly(I:C) synthetic dsRNA also upregulated the l(2)efl expression as high as $\sim 1.6$-fold, with a peak at 3 hpi. (C) DENV infection caused the phosphorylation of eukaryotic translation initiation factor 2, subunit 1 alpha (eIF2 $\alpha$ ). (D) Transfection with poly(I:C) and subsequent infection DENV resulted in higher phosphorylation levels up to $48 \mathrm{hpi}$. (E) Induction of $l(2)$ efl by poly(I:C) and the phosphorylation of eIF2 $\alpha$ reduced the DENV type 2 replication at 24 and $48 \mathrm{hpi}$. All the experiments were performed using three biological replicates (except for Figure D, which had two biological replicates). NI: noninfected; PIC: poly(I:C); DV: dengue virus. ${ }^{*} p<0.05$, ** $p<0.01$.

l(2)efl overexpression is known to result in the phosphorylation of eukaryotic translation initiation factor 2, subunit 1 alpha (eIF2 $\alpha$ ) in the fruit fly Drosophila melanogaster [29]. We observed a similar result in A. aegypti following DENV-2 infection, as eIF2 $\alpha$ was phosphorylated at serine position 51 (Figure 2C). The introduction of poly(I:C) also enhanced phosphorylation in cells infected with DENV-2 (Figure 2D). The eIF $2 \alpha$ phosphorylation may decrease protein synthesis and may also repress DENV-2 replication. We confirmed this predicted correlation and observed that the l(2)efl overexpression suppressed the DENV-2 replication by approximately 10-fold in poly(I:C)-treated CCL-125 cells at $48 \mathrm{~h}$ post infection (hpi) (Figure 2E).

\subsection{Suppression of L(2)efl-1 and L(2)efl-4 Enhances DENV-2 Replication}

The introduction of $l(2)$ efl- 1 dsRNAs into CCL-125 cells resulted in a $60 \%$ reduction in the transcript abundance up to $72 \mathrm{~h}$ post dsRNA-transfection (Figure 3A). However, the reduction in specific transcripts did not lead to any difference in DENV-2 titer at 24 and 48 hpi (Figure 3B). 


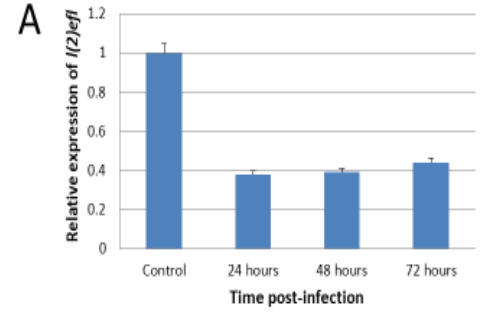

B

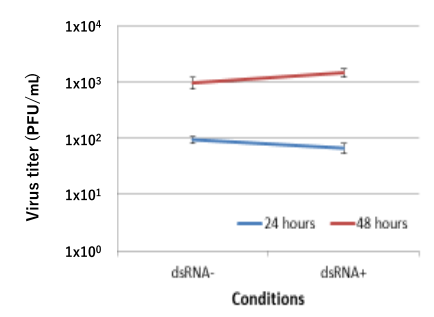

C

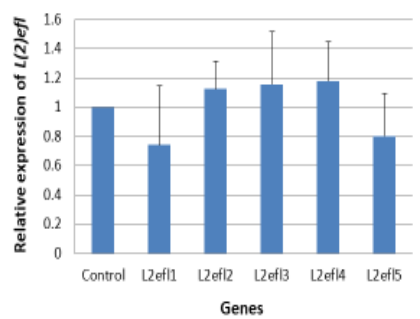

D

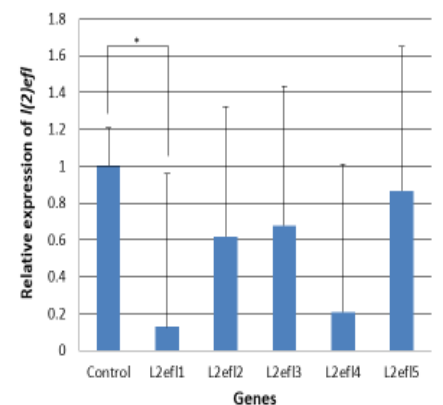

$E$

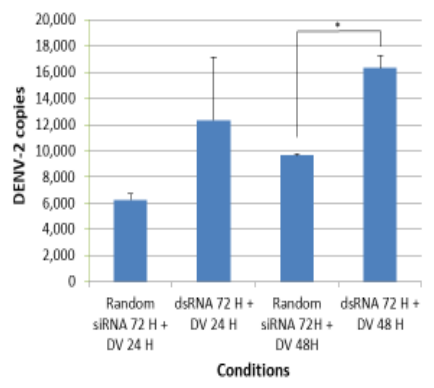

Figure 3. Suppression of $l(2)$ efl- 1 and $l(2)$ efl- 4 promotes dengue virus type 2 replication in CCL-125 cells. (A) Gene-specific dsRNAs ablated $60 \%$ of $l(2) e f l$ transcripts for as long as $72 \mathrm{~h}$ post transfection. (B) Suppression did not cause any difference in the dengue virus type 2 (DENV2) replication 24 and $48 \mathrm{~h}$ post infection, which may have been due to compensation by other l(2)efl homologs (C). (D) RNAi effectively repressed $l(2)$ efl-1 and $l(2)$ efl-4 transcripts, but only partially affected $l(2)$ efl-2 and $l(2) e f l-3$. (E) $l(2)$ efl- 1 and $l(2)$ efl- 4 transcript ablation enhanced the DENV2 replication by as much as 2 -fold in CCL-125 cells. All the experiments were performed using three biological replicates (except for Figures $B$ and $C$, which have two biological replicates). Rdm: random siRNA; dsRNA: double-stranded RNA; DV: dengue virus; H: hours. ${ }^{*} p<0.05$.

We hypothesized that the failure to observe an impact on the viral titer in the previous experiments may have resulted from compensation by other l(2)efl family members. Our RNA-seq data showed that there were multiple upregulated $l(2)$ efl homologs (Table 3$)$. We randomly checked another four $l(2)$ efl homologs and found that, after the introduction of $l(2)$ efl- 1 dsRNA, $l(2)$ ef $l-2,3$, and 4 were induced but not l(2)efl-5 (Figure 3C). This induction may have compensated for the l(2)efl-1 loss of action. Therefore, we suppressed the expression of $l(2)$ efl- 1 to $l(2)$ efl-4. dsRNA targeting these gene products effectively reduced the $l(2)$ efl- 1 and $l(2)$ efl- 4 transcripts, but it only partially reduced the abundance of $l(2) e f l-2$ and l(2)efl-3 (Figure 3D). l(2)efl-1- and l(2)efl-4-suppressed CCL-125 cells infected with DENV-2 had higher DENV-2 copies at 24 and 48 hpi (Figure 3E). We conclude that $l(2) e f l$ helps to suppress the DENV-2 replication in A. aegypti, but that one l(2)efl homolog alone is not enough to suppress virus replication and function.

\section{Material and Methods}

\subsection{Mosquito Rearing}

The Aedes aegypti LVP INB strain was grown at $26 \pm 1{ }^{\circ} \mathrm{C}$ and at $80-95 \%$ relative humidity, with a 16:8 h light:dark cycle. Larvae were fed on a combination of finely ground mouse food (CE-2, CLEA, Shizuoka, Japan), dried yeast EBIOS (\#2332001X1084, Asahi Food and Healthcare, Shibuya, Tokyo, Japan), and finely ground fish food (TetraMin, Spectrum Brands Japan, Yokohama, Kanagawa, Japan). 
Male and female adults were housed together in a cage with unlimited access to a $4 \%$ sugar solution until infection. The mosquitoes used in the experiments were 5-9 days post eclosion. Females were not blood fed prior to the experiments.

\subsection{Mosquito Infection}

The stock virus of DEN-2 ThNH7/93 was prepared by infecting C6/36 mosquito cells. Mosquitoes were infected with dengue virus as previously described (Runtuwene et al. 2014). Briefly, K562 erythroleukemia cells were infected with $1.5 \times 10^{7} \mathrm{PFU} / \mathrm{mL}$ DEN-2 ThNH7/93 (MOI 1.0) and then the cells were supplemented with D4-I-1D6 monoclonal antibody. The virus concentration in the mouse blood was maintained at $10^{4}$ to $10^{5} \mathrm{PFU} / \mathrm{mL}$ for at least up to $7 \mathrm{~h}$ post injection. Five- to nine-day-old female mosquitoes were allowed to blood-feed from the infected mice $5 \mathrm{~h}$ post-injection [21]. After blood feeding, the mosquitoes were incubated at $28^{\circ} \mathrm{C}$ for $2,6,10$, and 14 days. Both the infected group and the noninfected group were provided unlimited access to a cotton pad impregnated with a $4 \%$ sugar solution. All the mosquitoes completely digested the blood meal by 14 days post blood feeding. The mosquitoes were frozen for at least $1 \mathrm{~h}$ at $-80^{\circ} \mathrm{C}$ and stored at $-80^{\circ} \mathrm{C}$ until processing.

\subsection{Confirmation of Mosquito Infection}

Successful infection was confirmed using reverse transcriptase polymerase chain reaction (RT-PCR) with SuperScript III One-Step RT-PCR (Invitrogen, Carlsbad, CA, USA) and previously published universal DENV primers [30]. The DENV-2 titer in mosquitoes were measured with the plaque assay described below. The dissemination rate was determined by checking isolated mosquito legs for the DENV-2 genome. A positive sample indicated that the mosquito did not have midgut-restricted infection.

\subsection{RNA Extraction and Illumina Library Preparation}

Total RNA was extracted using a combination of ISOGEN (Nippon Gene, Tokyo, Japan) and TruSeq Stranded mRNA Sample Preparation (Illumina, San Diego, CA, USA) from control mosquitoes and those 14 days post-infection (dpi) to DENV-2. Each group consisted of two pools of 25 females. A total of four pools (i.e., two from each group) were prepared as four single-read Illumina libraries according to the manufacturer's protocol. After library validation with a bioanalyzer, the ds-cDNA was run for 40 cycles on an Illumina Genome Analyzer.

\subsection{RNA-seq Data Analyses}

The assembled supercontig sequences (version AaegL3) of the A. aegypti genome and the GTF annotation file (ver. AaegL3.3) were downloaded from VectorBase [31]. The supercontigs were used as the reference genome sequence. Sequence reads were mapped to the reference genome using Bowtie v2.2 and then TopHat v2.1 [32] with the GTF file, a segment length of 12, and a segment mismatch of 1. The Cuffdiff program within the package Cufflinks v2.2.1 [33] was used to calculate the reads per kilobase per million reads (RPKM) of each gene and to identify genes with significant transcript abundance differences between two samples with a false discovery rate (FDR) of $0.01 \%$. The entire primary sequencing data are archived in the DDBJ Sequence Read Archive (DRA) as accession number DRA002522.

\subsection{Transfection and Infection of the A. aegypti Cell Line CCL-125 with Poly(I:C) and DENV-2}

The A. aegypti CCL-125 (ATC-10) cell line (purchased from American Type Culture Collection, Manassas, VA, USA) was transfected with $30 \mu \mathrm{g} / \mathrm{mL}$ poly(I:C) (Sigma-Aldrich, St. Louis, MO, USA) using polyethylenimine (PEI, Sigma-Aldrich, St. Louis, MO, USA) at a working concentration of $3 \mu \mathrm{g} / \mathrm{mL}$. Prior to transfection, the cells were washed and incubated with serum-free minimal essential medium (MEM, Sigma-Aldrich, St. Louis, MO, USA) to cause serum starvation. The transfected cells 
were incubated at $28{ }^{\circ} \mathrm{C}$ with $5 \% \mathrm{CO}_{2}$ for 3,6 , and $12 \mathrm{~h}$ before harvesting. CCL- 125 cells were also infected with the ThNH7/93 DENV-2 strain at a multiplicity of infection (MOI) of 5.0, followed by incubation at $28^{\circ} \mathrm{C}$ and $5 \% \mathrm{CO}_{2}$ for one hour. The cells were washed to remove unbound virus particles and then incubated for 6,12 , and $24 \mathrm{~h}$ in MEM containing 2\% fetal bovine serum (FBS, HyClone, Characterized Grade, Canadian Origin, Cytiva, Marlborough, MA, USA) before collection. To analyze the effect of the l(2)efl upregulation on DENV-2 replication, the CCL-125 cells were transfected with poly(I:C) and incubated for $1.5 \mathrm{~h}$. The cells were then infected with the ThNH7/93 DENV-2 strain at an MOI of 5.0 and incubated for 24 and $48 \mathrm{~h}$ before cell collection.

\subsection{Immunoblotting of EIF $2 \alpha$}

Poly(I:C)-treated or DENV-2-infected CCL-125 cells were collected and lysed using lysis buffer $(1 \mathrm{mM}$ of DTT and $1 \times$ protease inhibitor in HEPES-NaCl-Glycerol-Triton X 100 (HNTG) buffer). The cell lysates were run on a $10 \%$ polyacrylamide gel. The proteins were transferred to a membrane (Millipore Immobilon-P, Merck Millipore, Burlington, MA, USA), which was incubated with anti-eIF2 $\alpha$ (phospho S51) primary antibody (Abcam, Cambridge, UK) for $12 \mathrm{~h}$. The peroxidase-conjugated mouse anti-rabbit IgG (Jackson Laboratory, Bar Harbor, ME, USA) secondary antibody was reacted with horseradish peroxidase (HRP) substrate (Merck Millipore, Burlington, MA, USA) Actin antibody (Jackson Laboratory, Bar Harbor, ME, USA) was used as a loading control.

\subsection{L(2)efl Suppression}

Four dsRNAs with sequences complementary to l(2)efl-1, l(2)efl-2, l(2)efl-3, and l(2)efl-4 (Supplementary Tables S1-S3) were synthesized using the MegaScript RNAi Kit (Ambion, Austin, TX, USA). Suppression was achieved by introducing $1 \mu \mathrm{g}$ of dsRNA directly to confluent CCL-125 cells. The cells were then incubated at $28{ }^{\circ} \mathrm{C}$ and $5 \% \mathrm{CO}_{2}$ for 24,48 , and $72 \mathrm{~h}$. Control samples were transfected with MISSION siRNA Universal Negative Controls (Sigma-Aldrich, St. Louis, MO, USA). To analyze the effect of $l(2)$ efl suppression on DENV-2 replication, CCL-125 cells were transfected with $l(2)$ efl-1, $l(2)$ efl-2, $l(2)$ efl-3, and $l(2)$ efl- 4 dsRNAs and incubated for $24 \mathrm{~h}$ prior to infection with the ThNH7/93 DENV-2 strain at an MOI of 5.0. The cells were incubated for 24 and $48 \mathrm{~h}$ before harvesting.

\subsection{Virus Titer}

Virus titer was measured with plaque assays. Briefly, mosquitoes or mosquito legs were homogenized in MEM containing 2\% FBS or supernatants of DENV-2-infected CCL-125 cells were collected, diluted to 10 to $10^{6}$-fold, and introduced to each well of a 12-well Corning plate (Corning Inc., Corning, NY, USA) layered with a confluent Vero cell monolayer. Following $2 \mathrm{~h}$ of incubation at $37^{\circ} \mathrm{C}$ and $5 \% \mathrm{CO}_{2}$ with occasional shaking every 5-10 min, the infected monolayer was layered with methylcellulose containing $2 \% \mathrm{FBS}$ and incubated again at $37^{\circ} \mathrm{C}$ and $5 \% \mathrm{CO}_{2}$ for 9 days. The overlay medium was removed, and the monolayer was fixed with formalin and stained with $1.25 \%$ methylene blue (Sigma-Aldrich, St. Louis, MO, USA). Plaques were counted, and the results were expressed as PFU per mosquito or PFU/mL.

\subsection{Quantitative RT-PCR}

A total of 10 genes identified by RNA-seq to be accumulated differentially between uninfected and infected mosquitoes were selected for real-time quantitative RT-PCR (qRT-PCR) analysis. Total RNA from a pool of either 10 uninfected or 10 infected females was extracted using a combination of TRIZOL reagent (Invitrogen, Carlsbad, CA, USA) and PureLink RNA Mini Kits (Ambion, Austin, TX, USA). qRT-PCR was performed using One Step SYBR PrimeScript PLUS (Takara Bio, Shiga, Japan) and a LightCycler 96 (Roche, Basel, Switzerland) (see Supplementary Tables S1-S3 for the primer sequences). Fold changes in transcript abundance between uninfected and infected mosquitoes were derived by the comparative CT method [34] using the 40srps17 gene (AAEL004715, which encodes the $40 \mathrm{~S}$ ribosomal protein 17) as the reference. We compared the stability of three housekeeping genes 
(40srps17 (AAEL004715), actin (AAEL004631), and $r p L 5$ (gi|94468377)) and found that 40srps17 was the most stable among them (Supplementary Figure S1). The correlation between the abundance values detected by RNA-seq and qRT-PCR for the 10 genes tested was estimated by calculating the Spearman Rho's R using online software [35]. The l(2)efl-1 to l(2)efl-5 abundance levels were also measured after the collection of poly(I:C)-transfected and DENV-2-infected CCL-125 cells using the same procedure (see Supplementary Tables S1-S3 for primer sequences). DENV-2 copies were measured by absolute quantification that required two qRT-PCR steps. cDNAs were produced using the Transcriptor First-Strand cDNA Synthesis Kit (Roche) using random primers. Measurement with qRT-PCR was performed using the LightCycler FastStart DNA Master SYBRGreen I (Roche, Basel, Switzerland) and a LightCycler 96. The DENV-2 copy standard was produced by Nihon Gene Research Laboratory Inc. (Sendai, Miyagi, Japan). Statistical significance was calculated using a Student's $t$-test.

\subsection{Ethics Statement}

The Committee for Animal Experiments at Oita University approved the experiments under permission numbered D00902. The Committee for Animal Experiments at Oita University complies with the Guidelines for Proper Conduct of Animal Experiments stipulated by the Science Council of Japan.

\section{Conclusions}

We observed complex changes in the A. aegypti transcriptome at 14 days after dengue virus infection. We identified at least one gene family-namely, lethal(2)-essential-for-life-to have a role in DENV-2 infection. Heat-shock proteins are induced during stress and act as chaperones to guide misfolded proteins [28]. The l(2)efl genes belong to the small HSP20 family, and while many family members are induced in the presence of stresses, $l(2) \mathrm{efl}$ transcription is upregulated by flavivirus infection [36], but not pollutants and insecticides [37]. When A. aegypti larvae are exposed to pollutants and insecticides as stressors, the l(2)efl gene is not upregulated [37], supporting the hypothesis that flaviviruses may be a selective upregulator of l(2)efl transcription. The overexpression of l(2)efl in D. melanogaster leads to the phosphorylation of eIF2 $\alpha$ [29]. This phosphorylation inhibits the formation of translation initiation complexes, leading to translation inhibition, a mechanism that is postulated to impede virus protein translation [38,39]. L(2)efl may also work in A. aegypti through the same mechanism. However, it remains unclear how l(2)efl phosphorylates eIF2 $\alpha$, which is the limitation of our paper. We focused the analysis only on l(2)efl gene members. This analysis oversimplifies the complex interaction between proteins in the A. aegypti. Other decreased proteins, such as the Toll subunit in salivary glands, might play a bigger role in keeping a higher DENV-2 titer in the saliva [40]. We also only looked at the dynamics of DENV-2, which is another limitation of this paper.

Supplementary Materials: The following are available online at http://www.mdpi.com/1422-0067/21/20/7520/s1, Figure S1: The Ct value among different experimental conditions on different time points for housekeeping genes 40s rps17 (A), actin (B), and rpL5 (C)., Table S1: List of primer sequences for dsRNA production, Table S2: List of primer sequences for RNA-seq validation, Table S3: List of primer sequences for l(2)efl-2 to l(2)efl-5, Table S4: Upregulated genes at 14 days post dengue infection in Aedes aegypti, Table S5: Downregulated genes at 14 days post dengue infection in Aedes aegypti.

Author Contributions: Conceptualization, L.R.R., and Y.E.; methodology, T.K.; software, S.K.; validation, S.N., M.S., and Y.O.; formal analysis, L.R.R., S.K., K.H., and J.Y.; investigation, T.K., and Y.E.; resources, Y.E.; data curation, L.R.R., and S.K.; writing-original draft preparation, L.R.R., and S.K.; writing-review and editing, H.S., A.A.J. and Y.E.; supervision, V.D.P., and J.S.B.T.; project administration, T.T.; funding acquisition, C.S., T.T., and Y.E. All authors have read and agreed to the published version of the manuscript.

Funding: This work was partially supported by the research project for comprehensive measures against vector-borne viral infectious diseases that have possible autochthonous epidemics in the Research Program on Emerging and Re-emerging Infectious Diseases (H26-28, H29-31), by the Japan Agency for Medical Research and Development (AMED) under Grant Numbers JP19fk0108035 and JP20fk0108123, and by Japan Society for the Promotion of Science (JSPS) under Grants-in-Aid for Scientific Research (Kiban-C, \#23590803; Kiban-B, \#25300053). 
Acknowledgments: We thank Terumi Horiuchi, Keiko Toya, Kazumi Abe, Kiyomi Imamura, and Makiko Tosaka for their technical assistance and Yutaka Suzuki for his scientific support at the Department of Computational Biology and Department of Medical Genome Sciences, Graduate School of Frontier Sciences, University of Tokyo; Kaori Noguchi, Shinya Hidano, and Naganori Kamiyama at the Department of Infectious Disease Control, Faculty of Medicine, Oita University; Sumio Sugano and Kenta Nakai at the Institute of Medical Science, University of Tokyo; Ryuichiro Maeda at the Department of Basic Veterinary Medicine, Obihiro University of Agriculture and Veterinary Medicine; Raweewan Srisawat and Narumon Komoramisra at the Departments of Medical Entomology, Faculty of Tropical Medicine, Mahidol University; and Ichiro Kurane at National Institute of Infectious Diseases, Tokyo for their scientific support. We also thank Mihoko Imada at the Department of Infectious Diseases, Keio University School of Medicine; the late Junichi Watanabe of the Institute of Medical Science, University of Tokyo; and Johnny Runtuwene and Arthur E. Mongan at the Faculty of Medicine, Sam Ratulangi University, for their scientific and moral support.

Conflicts of Interest: The authors declare no conflict of interest.

\section{References}

1. Gubler, D.J. Epidemic dengue/dengue hemorrhagic fever as a public health, social and economic problem in the 21st century. Trends Microbiol. 2002, 10, 100-103. [CrossRef]

2. World Health Organization. Dengue: Guidelines for Diagnosis, Treatment, Prevention and Control; WHO: Geneva, Switzerland, 2009.

3. Gubler, D.J. Dengue and dengue hemorrhagic fever. Clin. Microbiol. Rev. 1998, 11, 480-496. [CrossRef] [PubMed]

4. Sabchareon, A.; Wallace, D.; Sirivichayakul, C.; Limkittikul, K.; Chanthavanich, P.; Suvannadabba, S.; Jiwariyavej, V.; Dulyachai, W.; Pengsaa, K.; Wartel, T.A.; et al. Protective efficacy of the recombinant, live-attenuated, CYD tetravalent dengue vaccine in Thai schoolchildren: A randomised, controlled phase $2 \mathrm{~b}$ trial. Lancet 2012, 380, 1559-1567. [CrossRef]

5. Bhatt, S.; Gething, P.W.; Brady, O.J.; Messina, J.P.; Farlow, A.W.; Moyes, C.L.; Drake, J.M.; Brownstein, J.S.; Hoen, A.G.; Sankoh, O.; et al. The global distribution and burden of dengue. Nature 2013, 496, $504-507$. [CrossRef]

6. Del Angel, R.M.; Valle, J.R. Dengue vaccines: Strongly sought but not a reality just yet. PLoS Pathog 2013, 9, e1003551. [CrossRef]

7. World Health Organization. Updated Questions and Answers Related to Information Presented in the Sanofi Pasteur Press Release on 30 November 2017 with Regards to the Dengue Vaccine Dengvaxia ${ }^{\circledR}$. Available online: http://www.who.int/immunization/diseases/dengue/q_and_a_dengue_vaccine_dengvaxia/en/ (accessed on 20 May 2018).

8. Rieter, P.; Gubler, D.J. Surveillance and control of urban dengue vectors. In Dengue and Dengue Hemorrhagic Fever; Gubler, D.J., Kuno, G., Eds.; CAB International: Wallingford, UK, 1997; pp. 425-462.

9. James, A.A. Gene drive systems in mosquitoes: Rules of the road. Trends Parasitol. 2005, 21, 64-67. [CrossRef]

10. Franz, E.A.W.; Sanchez-vargas, I.; Adelman, Z.N.; Blair, C.D.; Beaty, B.J.; James, A.A.; Olson, K.E. Engineering RNA interference-based resistance to dengue virus type 2 in genetically modified Aedes aegypti. Proc. Natl. Acad. Sci. USA 2006, 103, 4198-4203. [CrossRef]

11. Behura, S.K.; Gomez-Machorro, C.; Harker, B.W.; DeBruyn, B.; Lovin, D.D.; Hemme, R.R.; Mori, A.; Romero-Severson, J.; Severson, D.W. Global cross-talk of genes of the mosquito Aedes aegypti in response to dengue virus infection. PLoS Negl. Trop. Dis. 2011, 5, e1385. [CrossRef] [PubMed]

12. Bonizzoni, M.; Dunn, W.A.; Campbell, C.L.; Olson, K.E.; Marinotti, O.; James, A.A. Complex modulation of the Aedes aegypti transcriptome in response to dengue virus infection. PLoS ONE 2012, 7, e50512. [CrossRef]

13. Guo, X.; Xu, Y.; Bian, G.; Pike, A.D.; Xie, Y.; Xi, Z. Response of the mosquito protein interaction network to dengue infection. BMC Genom. 2010, 11, 380. [CrossRef]

14. Doolittle, M.J.; Gomez, S.M. Mapping protein interactions between dengue virus and its human and insect hosts. PLoS Negl. Trop. Dis. 2011, 5, e954. [CrossRef]

15. Lee, H.L.; Wong, Y.C.; Rohani, A. Protein profiles of dengue-infected Aedes aegypti (L). Dengue Bull. 2009, 33, $115-123$.

16. Sim, S.; Dimopoulos, G. Dengue virus inhibits immune responses in Aedes aegypti cells. PLoS ONE 2010, 5, e10678. [CrossRef] 
17. Barón, O.L.; Ursic-Bedoya, R.J.; Lowenberger, C.A.; Ocampo, C.B. Differential gene expression from midguts of refractory and susceptible lines of the mosquito, Aedes aegypti, infected with dengue-2 virus. J. Insect Sci. 2010, 10, 1-23. [CrossRef]

18. Sim, S.; Ramirez, J.L.; Dimopoulos, G. Dengue virus infection of the Aedes aegypti salivary gland and chemosensory apparatus induces genes that modulate infection and blood-feeding behavior. PLoS Pathog. 2012, 8, e1002631. [CrossRef]

19. Xi, Z.; Ramirez, J.L.; Dimopoulos, G. The Aedes aegypti toll pathway controls dengue virus infection. PLoS Pathog. 2008, 4, e1000098. [CrossRef]

20. Souza-Neto, J.A.; Sim, S.; Dimopoulos, G. An evolutionary conserved function of the JAK-STAT pathway in anti-dengue defense. Proc. Natl. Acad. Sci. USA 2009, 106, 17841-17846. [CrossRef]

21. Runtuwene, L.R.; Konishi, E.; Yamanaka, A.; Makino, Y.; Suzuki, Y.; Takasaki, T.; Kurane, I.; Kobayashi, T.; Eshita, Y. Dengue transmission model by means of viremic adult immuno-competent mouse. Parasit. Vectors 2014, 7, 143. [CrossRef]

22. Nene, V.; Wortman, J.R.; Lawson, D.; Haas, B.; Kodira, C.; Tu, Z.J.; Loftus, B.; Xi, Z.; Megy, K.; Grabherr, M.; et al. Genome sequence of Aedes aegypti, a major arbovirus vector. Science 2007, 316, 1718-1723. [CrossRef]

23. Leitmeyer, K.C.; Vaughn, D.W.; Watts, D.M.; Salas, R.; Villalobos, I.; de Chacon; Ramos, C.; Rico-Hesse, R.; de Chachon, I.V.; Ramos, C.; et al. Dengue virus structural differences that correlate with pathogenesis. J. Virol. 1999, 73, 4738-4747. [CrossRef]

24. Pandey, B.D.B.; Morita, K.; Hasebe, F.; Parquet, M.; Igarashi, A.; Parquet, M.C.; Igarashi, A. Molecular evolution, distribution and genetic relationship among the dengue 2 viruses isolated from different clinical severity. Southeast Asian J. Trop. Med. Public Health 2000, 31, 266-272.

25. Salazar, M.I.; Richardson, J.H.; Sánchez-Vargas, I.; Olson, K.E.; Beaty, B.J. Dengue virus type 2: Replication and tropisms in orally infected Aedes aegypti mosquitoes. BMC Microbiol. 2007, 7, 9. [CrossRef]

26. The Gene Ontology Consortium. Gene ontology: Tool for the unification of biology. Nat. Genet. 2000, 25, 25-29. [CrossRef]

27. Colpitts, T.M.; Barthel, S.; Wang, P.; Fikrig, E. Dengue virus capsid protein binds core histones and inhibits nucleosome formation in human liver cells. PLoS ONE 2011, 6, e24365. [CrossRef]

28. Zhao, L.; Pridgeon, J.W.; Becnel, J.J.; Clark, G.G.; Linthicum, K.J. Identification of genes differentially expressed during heat shock treatment in Aedes aegypti. J. Med. Entomol. 2009, 46, 490-495. [CrossRef]

29. Carra, S.; Boncoraglio, A.; Kanon, B.; Brunsting, J.F.; Minoia, M.; Rana, A.; Vos, M.J.; Seidel, K.; Sibon, O.C.M.; Kampinga, H.H. Identification of the Drosophila ortholog of HSPB8: Implication of HSPB8 loss of function in protein folding diseases. J. Biol. Chem. 2010, 285, 37811-37822. [CrossRef]

30. Morita, K.; Maemoto, T.; Honda, S.; Onishi, K.; Murata, M.; Tanaka, M.; Igarashi, A. Rapid detection of virus genome from imported dengue fever and dengue hemorrhagic fever patients by direct polymerase chain reaction. J. Med. Virol. 1994, 44, 54-58. [CrossRef]

31. Lawson, D.; Arensburger, P.; Atkinson, P.; Besansky, N.J.; Bruggner, R.V.; Butler, R.; Campbell, K.S.; Christophides, G.K.; Christley, S.; Dialynas, E.; et al. VectorBase: A data resource for invertebrate vector genomics. Nucleic Acids Res. 2009, 37, 583-587. [CrossRef]

32. Trapnell, C.; Pachter, L.; Salzberg, S.L. TopHat: Discovering splice junctions with RNA-Seq. Bioinformatics 2009, 25, 1105-1111. [CrossRef]

33. Trapnell, C.; Williams, B.; Pertea, G.; Mortazavi, A.; Pachter, L.; van Baren, J.; Salzberg, S.L.; Wold, B.J.; Pachter, L. Transcript assembly and abundance estimation from RNA-seq reveals thousands of new transcripts and switching among isoforms. Nat. Biotechnol. 2010, 28, 511-515. [CrossRef]

34. Schmittgen, T.; Livak, K. Analyzing real-time PCR data by the comparative CT method. Nat. Protoc. 2008, 3, 1101-1108. [CrossRef] [PubMed]

35. Social Science Statistics. n.d. Spearman's Rho Calculator. Available online: http://www.socscistatistics.com/ tests/spearman/default2.aspx (accessed on 18 June 2014).

36. Colpitts, T.M.; Cox, J.; Vanlandingham, D.L.; Feitosa, F.M.; Cheng, G.; Kurscheid, S.; Wang, P.; Krishnan, M.N.; Higgs, S.; Fikrig, E. Alterations in the Aedes aegypti transcriptome during infection with West Nile, dengue and yellow fever viruses. PLoS Pathog. 2011, 7, e1002189. [CrossRef] 
37. David, J.-P.; Coissac, E.; Melodelima, C.; Poupardin, R.; Riaz, M.A.; Chandor-Proust, A.; Reynaud, S. Transcriptome response to pollutants and insecticides in the dengue vector Aedes aegypti using next-generation sequencing technology. BMC Genom. 2010, 11, 216. [CrossRef]

38. Williams, B.R.G. PKR; a sentinel kinase for cellular stress. Oncogene 1999, 18, 6112-6120. [CrossRef]

39. Connor, J.H.; Lyles, D.S. Inhibition of host and viral translation during vesicular stomatitis virus infection: eIF2 is responsible for the inhibition of viral but not host translation. J. Biol. Chem. 2005, 280, 13512-13519. [CrossRef]

40. Chisenhall, D.M.; Londono, B.L.; Christofferson, R.C.; McCracken, M.K.; Mores, C.N. Effect of Dengue-2 Virus in the Salivary Glands of Aedes aegypti Mosquitoes. Am. J. Trop. Med. Hyg. 2014, 90, 431-437. [CrossRef]

(C) 2020 by the authors. Licensee MDPI, Basel, Switzerland. This article is an open access article distributed under the terms and conditions of the Creative Commons Attribution (CC BY) license (http://creativecommons.org/licenses/by/4.0/). 